\title{
REFLEXÕES ACERCA DO ATRIBUTO ORIENTAÇ̃̃O COMUNITÁRIA NAS ESTRATÉGIAS SAÚDE DA FAMÍLIA DE SANTA MARIA
}

\author{
Lorena Alves Fiorenza1; Ânderson Rosauro Eich²; Naiana Oliveira dos Santos³
}

\section{RESUMO}

A Atenção Primária em Saúde é responsável pela coordenação e integração do cuidado e possui atributos essenciais e derivados que podem ser avaliados por meio de uma ferramenta chamada PCATool-Brasil. O objetivo da pesquisa é apresentar a questão que foi mais bem avaliada do atributo orientação comunitária na percepção de enfermeiros das ESF do município de Santa Maria/ Rio Grande do Sul. Estudo descritivo, transversal de abordadem quantitativa. Utilizou-se um formulário online com questões do PCATool-Brasil referentes ao atributo em questão, a análise foi feita a partir da média aritimética das questões e depois aplicada uma fórmula para transformar em escala de 0 a 10. O escore de orientação comunitária foi 7,48 considerado acima da média $(>6,6)$. As visitas domiciliares (VD) são pontos fortes desse atributo na cidade e corroboram com achados em pesquisas de outros autores. Espera-se que esse estudo estimule a realização das VD pelos profissionais de saúde.

Palavras-chave: Atenção Primária em Saúde; Enfermagem; Pesquisa.

Eixo Temático: Atenção Integral e Promoção à Saúde (AIPS).

\section{INTRODUÇÃO}

A Atenção Primária em Saúde (APS) é configurada como porta de entrada do Sistema Único de Saúde (SUS) e exerce funções de coordenação e integração do cuidado. Além disso, a APS tem sua estratégia de atuação focada nas Unidades Básicas de Saúde (UBS) e Estratégias Saúde da Família (ESF). Essas visam ampliar a promoção e prevenção de saúde, além de buscar ações de recuperação que sejam resolutivas e eficazes, tendo o seu cuidado centrado na população e nas

\footnotetext{
${ }^{1}$ Acadêmica do curso de graduação em enfermagem - Universidade Franciscana. E-mail: lorenafiorenzza@gmail.com

2 Acadêmico do curso de graduação em enfermagem - Universidade Franciscana. E-mail: anderson eich1@hotmail.com

${ }^{3}$ Professora Doutora em enfermagem - Universidade Franciscana. E-mail: naiana.santos@ufn.edu.br
} 
demandas da comunidade (MACHADO et al., 2021).

Os atributos da APS são divididos em duas categorias, sendo elas os atributos essenciais (acesso ao primeiro contato, longitudinalidade, coordenação da atenção e integralidade) e atributos derivados (orientação familiar, orientação comunitária e competência cultural). A análise desses atributos é feita por meio de um instrumento reconhecido a nível mundial e adaptado para a realidade brasileira denominado PCATool-Brasil. Essa ferramenta conta com três versões, uma para os usuários adultos, outra para usuários infantis e a última para profissionais e/ou gestores de saúde (ABRANTES et al., 2020).

As Estratégias Saúde da Família, anteriormente chamadas de Programa Saúde da Família (PSF), foram consideradas um marco histórico no que tange a APS do Brasil. Instaurado em 1994, o PSF se tornou o principal meio para a ampliação do acesso ao primeiro contato e para a reorganização dos serviços de saúde através de uma mudança no modelo assistencial. O PSF previa um cuidado centrado nas famílias e comunidades, além da atuação de uma equipe multiprofissional com agentes comunitários de saúde (ACS) no intuito de promover o cuidado integral e longitudinal (PINTO; GIOVANELLA, 2018) (RECIHERT et al., 2016).

O atributo Orientação Comunitária, foco desse estudo, refere-se ao reconhecimento das necessidades em saúde da comunidade através da análise de dados epidemiológicos, do contato do serviço de saúde com a população e o planejamento e a avaliação conjunta dos serviços (BRASIL, 2020). Sendo assim, a APS, com base na orientação comunitária, deve utilizar os dados epidemiológicos no intuito de promover uma atenção com enfoque nas prioridades daquela comunidade, oferecendo ações de saúde diferentes de outra região que possui outras demandas (PAULA et al., 2017).

O Agente Comunitário de Saúde (ACS) tem no seu trabalho a consolidação dos atributos orientação comunitária e competência cultural, pois é o profissional que, através das visitas domiciliares (VD), se relaciona diariamente com as famílias do seu território e transita entre os saberes técnicos e populares, aumentando assim a criação de vínculos entre serviço e comunidade (MACIEL et al., 2020). 
Porém, um trabalho evidenciou que as VD's estão, ao longo do tempo, se tornando cada vez mais escassas, apesar do aumento da população cadastrada. Os autores apontam que esse problema pode ter relação com a ausência dos moradores no horário da visita e condomínios que impossibilitam a entrada do ACS. Ainda, identificaram que a intensificação de atividades dentro dos serviços, no acolhimento e preenchimento de formulários, além da entrega da referência de exames e consultas especializadas agendadas, podem ter relação com a baixa quantidade de visitas domiciliares realizadas (GIOVANELLA et al., 2021).

Apesar da forte discussão acerca da importância de a APS ser voltada à comunidade, outro estudo mostrou que alguns artigos feitos com vistas para 0 atributo de orientação comunitária possuíram escores abaixo da média $(<6,6)$. Segundos os autores, para que os serviços de saúde orientados à comunidade tenham sucesso, é necessário mais interação e participação popular, com um aumento de pesquisas locais para a delimitação das exigências das comunidades. Ainda, se faz necessário o desenvolvimento de estratégias de gestão participativas que trabalhem as situações mais urgentes, buscando as intervenções mais efetivas e que sejam modificadas e reorganizadas ao longo do tempo (BRUNELLI et al., 2021).

O presente artigo possui como objetivo apresentar a questão que foi mais bem avaliada do atributo orientação comunitária na percepção de enfermeiros das ESF do município de Santa Maria/ Rio Grande do Sul.

\section{METODOLOGIA}

Trata-se de um estudo descritivo, transversal de abordagem quantitativa, realizado no período de março a maio de 2021, na cidade de Santa Maria, Rio Grande do Sul, Brasil. A cobertura das ESF no período de coleta de dados era de 29,35\% da população, contando com 24 equipes de Estratégia Saúde da Família (BRASIL, 2020a). Este estudo faz parte do projeto de pesquisa denominado "Atributos da atenção primária à saúde na percepção de enfermeiros das Estratégias de Saúde da Família no município de Santa Maria - RS" e conta com o parecer do Comitê de Ética e Pesquisa (CEP) número 4.364.738. 
Ao todo, 24 enfermeiros das ESF da cidade de Santa Maria foram convidados a participar da pesquisa. O critério de inclusão foi estar na equipe há, no mínimo, seis meses, já que o tempo é um fator importante para a criação de vínculos entre os profissionais e usuários. Como critério de exclusão, adotou-se estar afastado por motivo de férias, licença médica ou afastamento do serviço.

Foi realizado um contato prévio por meio do telefone fixo das ESF no intuito de solicitar os e-mails dos enfermeiros atuantes nos serviços. Logo, foi encaminhado para esses e-mails, um link que dava acesso à plataforma GoogleForms, onde estavam presentes o Termo de Consentimento Livre e Esclarecido (TCLE), um questionário sociodemográfico e o Instrumento de Avaliação da Atenção Primária à Saúde (PCATool - Brasil) para profissionais médicos e enfermeiros versão extensa.

A avaliação sociodemográfica visava conhecer a experiência profissional e acadêmica das profissionais, contando com variáveis independentes o sexo (feminino e masculino), idade (em anos), tempo de formação (em anos), escolaridade (superior completo, especialização ou mestrado) e se o profissional possuía mais de um vínculo empregatício.

Como tempo de resposta inicial para a pesquisa online, foi considerado 15 dias. Devido à baixa adesão dos participantes para responder as questões, foram realizados mais dois contatos via telefone para os serviços de saúde. O período para a resposta do questionário foi estendido para um prazo de 30 dias. Estimou-se 30 minutos como o tempo para a resposta do instrumento.

O PCATool possui o objetivo de avaliar a Atenção Primária em saúde através da avaliação dos atributos essenciais (acesso ao primeiro contato, longitudinalidade, coordenação da atenção e integralidade) e atributos derivados (orientação familiar, orientação comunitária e competência cultural). Os atributos que fazem parte do instrumento podem ser avaliados em conjunto ou separadamente. Para este estudo, foi analisado o atributo de Orientação Comunitária que, no instrumento, é referenciado com a letra $\mathrm{H}$ e conta com 21 questões referentes a ele (BRASIL, 2020b).

QUADRO 1 - QUESTÕES REFERENTES AO ATRIBUTO ORIENTAÇÃO COMUNITÁRIA 
H1. Você ou alguém do seu serviço de saúde faz visitas domiciliares?

H2. Você acredita que o seu serviço de saúde tem o conhecimento adequado dos problemas de saúde da comunidade atendida?

H3. No seu serviço de saúde são obtidas opiniões e ideias da comunidade sobre como melhorar os serviços de saúde?

H4. No seu serviço de saúde podem ser adaptados serviços ou programas em resposta a problemas específicos de saúde da comunidade?

No seu serviço de saúde, os seguintes tipos de dados são utilizados para determinar quais os programas ou serviços são necessários à comunidade atendida?

H5. Informações de mortalidade (dados sobre óbitos)

H6. Dados de doenças de notificação compulsória (ex.: DSTs, TB).

H7. Taxas de imunização da comunidade.

H8. Dados secundários sobre saúde e riscos ocupacionais.

H9. Informações clínicas do próprio serviço (ex.: número de gestantes, número de pacientes hipertensos, número de pacientes com TB).

\section{No seu serviço de saúde os seguintes métodos são utilizados para monitorar e/ou avaliar a} efetividade dos serviços ou programas?

H10. Pesquisas com os seus pacientes.

H11. Pesquisas na sua comunidade.

H12. Feedback (retorno das informações) de organizações comunitárias ou conselhos gestores de saúde.

H13. Feedback (retorno das informações) da equipe de saúde.

H14. Análise de dados de saúde locais ou estatísticas vitais.

H15. Avaliações sistemáticas de seus programas e serviços prestados.

H16. Atuação dos Agentes Comunitários de Saúde.

H17. Presença de usuários no Conselho Local de Saúde (Conselho Gestor/Conselho de Usuários) ou Conselho Distrital de Saúde.

No seu serviço de saúde as seguintes atividades são utilizadas para alcançar as populações da comunidade atendida?

H18. Atuar em rede com agências estatais e locais envolvidas com grupos culturalmente diversos.

H19. Vínculos com serviços/organizações religiosas.

H20. Envolvimento com associações de moradores/lideranças comunitárias.

H21. Agentes comunitários de saúde ou membros do Conselho Local de Saúde (Conselho Gestor/Conselho de Usuários) ou Conselho Distrital de Saúde.

Fonte: Brasil, 2020b 
As respostas para cada item do PCATool-Brasil, são baseadas na escala Likert: "com certeza sim" (valor=4); "provavelmente sim" (valor=3); "provavelmente não" (valor=2); "com certeza não" (valor=1) e "não sei/não lembro" (valor=9). As respostas dos participantes ficaram armazenadas automaticamente em uma planilha na plataforma Google Drive. Após download dessa planilha, os dados foram transferidos para o Microsoft Excel para realização da análise estatística descritiva por meio de frequência absoluta e relativa e cálculo do escore.

O escore do atributo orientação comunitária, conforme o Manual do PCAToolBrasil, foi calculado pela média aritmética simples dos valores das respostas para cada pergunta e, posteriormente, transformado em escala contínua de 0 a 10 , utilizando a fórmula recomendada pelo manual: [(escore obtido-1) X 10] /3. Ainda, segundo o manual do PCATool-Brasil, o valor de referência para os escores é de 6,6 , sendo os abaixo desse valor $(<6,6)$ considerados baixa orientação da APS e os acima desse valor $(>6,6)$ alta orientação da APS, demonstrando efetividade e resolubilidade dos serviços (BRASIL, 2020b).

\section{RESULTADOS E DISCUSSÕES}

Ao todo, participaram da pesquisa 14 enfermeiras das 24 Estratégias Saúde da Família da cidade de Santa Maria, totalizando uma porcentagem de $100 \%$ na variável sexo feminino. Os outros 10 enfermeiros se recusaram a participar da pesquisa e/ou não foram encontrados nas tentativas de contato via telefone e/ou correio eletrônico (e-mail).

A idade das participantes variou entre 26 e 52 anos, sendo a maioria, com idade de 31 a 40 anos (42,85\%). No quesito formação, prevaleceram as participantes que possuíam especialização (35,71\%) ou mestrado $(57,14 \%)$. Acerca do tempo de atuação no serviço de saúde, parcela significativa da amostra atuava entre 2 e 10 anos (71,42\%). Sobre outros vínculos empregatícios, apenas três participantes $(21,4 \%)$ possuíam vínculo com outros serviços.

O atributo Orientação Comunitária alcançou um escore geral de 7,48, sendo considerado um atributo de alta orientação para a Atenção Primária em Saúde da cidade de Santa Maria. A questão "Você ou alguém do seu serviço de saúde faz 
visitas domiciliares?" foi a mais bem avaliada, trazendo um percentual de $100 \%$ das respostas "com certeza sim". Esse percentual alto de respostas positivas, se deve ao fato da pesquisa ter sido realizada com enfermeiras das ESF, compreendendo que a visita domiciliar é uma das ferramentas utilizadas nesses serviços de saúde.

Para que as visitas domiciliares (VD) na APS sejam executadas com qualidade, é necessário um planejamento prévio. Assim, as reuniões de equipe são a principal ferramenta para a realização de uma VD, pois é nela que os profissionais discutem os casos encontrados na comunidade e definem o papel de cada um. Nas reuniões de equipe deve ser feita uma estratificação dos usuários, definindo casos mais urgentes e aqueles que podem esperar, tendo em vista a grande demanda dos serviços de saúde. Além disso, é preciso verificar quais profissionais irão em cada VD e constatar a disponibilidade de todos, o horário das visitas, assim como o meio de locomoção de cada um e os materiais necessário para elas, além de definir o itinerário dos profissionais durante o turno das visitas (SAVASSI, 2016).

A visita domiciliar é, portanto, caracterizada como um instrumento muito rico que promove o aumento do vínculo serviço/comunidade e ainda permite coletar dados significativos para a realização das ações em saúde adequadas para aquela realidade. Além disso, é nas visitas domiciliares que se realiza a edudação em saúde, de modo que o profissional transmita seu conhecimento e permita que os usuários possam, da mesma forma, falar sobre seus saberes, estabelecendo assim, um conhecimento compartilhado, trazendo a comunidade para o centro de seu cuidado e fortalecendo a autonomia dos usuários (ALVES; ACIOLI, 2020).

Um estudo publicado no ano de 2017, buscou avaliar o atributo de orientação comunitária em três municípios do estado de Pernanbuco, pela visão de profissionais das ESF, em relação à saúde da criança. Nele a questão referente a realização de visitas domiciliares atingiu média máxima, representando a mesma realidade evidenciada na cidade de identificação de fatores ambientais e hábitos nocivos à saúde da criança, além de realizar ações de vigilância da saúde, idenificando vulnerabilidades e situações de risco (PAULA et al., 2017).

Outra pesquisa que abordava o atributo orientação comunitária foi realizada no estado do Rio Grande do Sul, contando com a participação de 25 municípios. 
Nele, o atributo apresentou escore mais na avaliação pelas ESF do que pelas Unidades Básicas de Saúde. Porém, as VD foram a única questão bem avaliada nesse estudo, sendo assim, os autores apontam que os profissionais de saúde, no planejamento de suas ações, considerem a opinião do usuário e seus familiares, para a realização de um cuidado voltado à comunidade (ANTUNES; PADOIN; PAULA, 2018).

Apesar dessa pesquisa apontar a realização das visitas domiciliares como um ponto positivo nessa localidade, é preciso compreender a qualidade dessas VD. Uma pesquisa que avaliou a realização da VD em território nacional, evidenciou que, muitas vezes, a estratificação dos usuários é muito focada na doença, geralmente as doenças crônicas não transmissíveis e a dificuldade de locomoção. Porém, questões como vulnerabilidade social e a verificação das condições ambientais no domicílio são deixadas em segundo plano (NUNES et al., 2018).

Ainda, corroborando com o escrito acima, muitos profissionais encaram a visita domiciliar como uma ferramenta burocrática e pouco relacional, preferindo o consultório como local de atuação, devido a alta vulnerabilidade dos usuários. Porém, entende-se que é no momento da realização da VD que se identificam as questões sociais e econômicas da popullação, pois estamos adentrando o local onde vivem essas pessoas. Além disso, o fato do profissional de saúde ir até o domicílio possibilita a ampliação de vínculos e de confiança entre os sujeitos, fazendo da VD, uma ferramenta essencial nos serviços de saúde (SANTOS; ROMANO; ENGSTROM, 2018).

\section{CONCLUSÃO}

O estudo concluiu que o atributo orientação comunitária obteve um escore acima da média na cidade de Santa Maria, demonstrando uma alta orientação para a Atenção Primária em Saúde. Ainda, verificou-se que as visitas domiciliares são realizadas de forma regular na cidade e que devem ser mantidas e estimuladas cada vez mais, pois tratam-se de instrumentos ricos para a construção de vínculo entre usuários e serviço de saúde, além de tornar a APS cada vez mais orientada à comunidade. 
Apesar da realização das visitas domiciliares serem feitas em todos os serviços na cidade de Santa Maria, sabe-se que não é a realidade encontrada em outras localidades. Ainda, entende-se que, muitas vezes, a VD é realizada apenas como burocracia, sendo deixados de lado a identificação de diversos fatores essenciais para a realização de ações prioritárias. Por esse motivo, espera-se que esse estudo inspire os profissionais de saúde a realizarem as VD com qualidade e eficiência, considerando a magnitude de benefícios trazidos por ela e, ainda, que cada vez mais nossos serviços de saúde se tornem resolutivos e orientados à comunidade.

\section{REFERÊNCIAS}

ABRANTES, R. S. de; MONTEIRO, D. L. A.; LUZ, A. P. R. G.; OlindA, R. A. de; PADILHA, W. W. N. Assessment of Primary Health Care Attributes in Campina Grande, Paraíba, Brazil. Revista Brasileira de Enfermagem, [S.L.], v. 73, n. 5, p. 18, 2020.2 Disponível em: https://www.scielo.br/j/reben/a/4PtrFfWD67tzy3QCrFXP6Bg/?lang=en. Acesso em: 13 set. 2021.

ALVES, L. V. V.; ACIOLI, S. Saberes científicos e populares de enfermeiros e usuários na visita domiciliar. Revista Cubana de Enfermería, [S. L.], v. 36 n. 3, p. 1 - 10, $2020 . \quad$ Disponível em: http://scielo.sld.cu/scielo.php?script=sci arttext\&pid=S0864-03192020000300012. Acesso em: 13 set. 2021

ANTUNES, B. S.; PADOIN, S. M. M.; PAULA, C. C. de. Avaliação dos atributos da atenção primária à saúde: criança e adolescente vivendo com HIV. Escola Anna Nery, [S. L.], v. 22, n. 2, p. 1-9, 2018. Disponível em: https://www.scielo.br/j/ean/a/hm7mbF9H7DgR7P7msTWc4ZG/?lang=pt. Acesso em: 16 set. 2021.

BRASIL. Ministério da Saúde. Pesquisa nacional de saúde - 2019: informações sobre domicílios, acesso e utilização dos serviços de saúde. IBGE: Rio de Janeiro, 2020a.

BRASIL. Ministério da Saúde. Secretaria de Atenção Primária à Saúde. Departamento de Saúde da Família. Manual do instrumento de avaliação da atenção primária à saúde: PCATool-Brasil. Brasília: Ministério da Saúde, 2020b. 237 p.

BRUNELLI, B.; LANGKAMER, M. F. B.; DORNELAS, A. J. S.; RODRIGUES, M. J. M.; BERNARDES, J. G. Orientação Comunitária: uma revisão integrativa. Revista Brasileira de Medicina de Família e Comunidade, [S.L.], v. 16, n. 43, p. 1-14, 30 maio 2021. Disponível em: https://rbmfc.org.br/rbmfc/article/view/2768. Acesso em: 13 set. 2021. 
GIOVANELLA, L.; BOUSQUAT, A.; SCHENKMAN, S.; ALMEIDA, P. F. de; SARDINHA, L. M. V.; VIEIRA, M. L. F. P. Cobertura da Estratégia Saúde da Família no Brasil: o que nos mostram as pesquisas nacionais de saúde 2013 e 2019. Ciência \& Saúde Coletiva, [S.L.], v. 26, n. 1, p. 2543-2556, jun. 2021. Disponível em: https://www.scielo.br/j/csc/a/SMZVrPZRgHrCTx57H35Ttsz/?lang=pt\&format=pdf\#: :t ext=Entre\%202013\%20e\%202019\%2C\%20observa,entre\%20cadastra\%20dos\%20na\%20USF. Acesso em: 14 set. 2021.

MACHADO, G. A. B.; DIAS, B. M.; SILVA, J. J.; BERNARDES, A.; GABRIEL, C. S. Avaliação de atributos da Atenção Primária à Saúde: a perspectiva dos profissionais. Acta Paulista de Enfermagem, [S.L.], v. 34, n. 00973, p. 1-8, 2021. Disponívem em: https://www.scielo.br/j/ape/a/zH64QjdJHyKjYRGMYky7h9j/. Acesso em: 14 set. 2021.

MACIEL, F. B. M.; SANTOS, H. L. P. C. dos; CARNEIRO, R. A. da S.; SOUZA, E. A. de; PRADO, N. M. B. L.; TEIXEIRA, C. F. S. Agente comunitário de saúde: reflexões sobre o processo de trabalho em saúde em tempos de pandemia de covid19. Ciência \& Saúde Coletiva, [S.L.], v. 25, n. 2, p. 4185-4195, out. 2020. Disponível em: https://www.scielo.br/j/csc/a/XsyXgfVksPRS38tgfYppqBb/?lang=pt. Acesso em: 15 set. 2021.

NUNES, C. A.; AQUINO, R.; MEDINA, M. G.; VILASBÔAS, A. L. Q.; PINTO JÚNIOR, E. P.; LUZ, L. A. da. Visitas domiciliares no Brasil: características da atividade basilar dos agentes comunitários de saúde. Saúde em Debate, [S.L.], v. 42, n. 2, p. 127144, out. 2018.2 Disponível em: https://www.scielo.br/j/sdeb/a/HL6TRrF7NyvpWjXdVyfhzsH/?lang=pt\&format=pdf. Acesso em: 17 set. 2021.

PAULA, W. K. A. S. de; SAMICO, I. C.; CAMINHA, M. F. C.; BATISTA FILHO, M.; FIGUEIRÔA, J. N. Orientação comunitária e enfoque familiar: avaliação de usuários e profissionais da estratégia saúde da família. Cadernos Saúde Coletiva, [S.L.], v. 25, n. 2, p. 242-248, 10 jul. 2017. Disponível em: https://www.scielo.br/j/cadsc/a/WJmKxBs9m3FRtDDx93VxRbF/abstract/?lang=pt. Acesso em: 13 set. 2021.

PINTO, L. F.; GIOVANELLA, Ligia. Do Programa à Estratégia Saúde da Família: expansão do acesso e redução das internações por condições sensíveis à atenção básica (ICSAB). Ciência \& Saúde Coletiva, [S.L.], v. 23, n. 6, p. 1903-1914, jun. 2018.

https://www.scielo.br/j/csc/a/dXV7f6FDmRnj7BWPJFt6LFk/abstract/?lang=pt. Acesso em: 13 set. 2021.

REICHERT, A. P. S.; LEÔNICO, A. B. A.; TOSO, B. R. G.; SANTOS, N. C. C. B.; VAZ, E. M. C.; COLLET, N. Orientação familiar e comunitária na Atenção Primária à Saúde da criança. Ciência \& Saúde Coletiva, [S.L.], v. 21, n. 1, p. 119-127, jan. 2016. Disponível em: https://www.scielosp.org/article/csc/2016.v21n1/119-127/. Acesso em: 15 set. 2021. 
SANTOS, R. O. M. dos; ROMANO, V. F.; ENGSTROM, E. M.. Vínculo longitudinal na Saúde da Família: construção fundamentada no modelo de atenção, práticas interpessoais e organização dos serviços. Physis: Revista de Saúde Coletiva, [S.L.], v. $28, \quad$ n. 2, p. 1-18, 13 ago. 2018. Disponível em: https://www.scielo.br/j/physis/a/GNjxJkJFNrHNxGVBNSdjMFJ/?format=pdf\&lang=pt. Acesso em: 17 set. 2021.

SAVASSI, L. C. M. Os atuais desafios da Atenção Domiciliar na Atenção Primária à Saúde: uma análise na perspectiva do sistema único de saúde. Revista Brasileira de Medicina de Família e Comunidade, [S.L.], v. 11, n. 38, p. 1-12, 17 ago. 2016. Disponível em: https://rbmfc.org.br/rbmfc/article/view/1259. Acesso em: 16 set. 2021. 